\title{
As condutas de enfermagem frente ao incentivo do homem no pré-natal: um estudo bibliográfico
}

\author{
Nursing conducts in view of men's encouragement in prenatal care: a bibliographic study \\ Conductas de enfermería en vista del estímulo de los hombres en la atención prenatal: un estudio \\ bibliográfico
}

Recebido: 20/11/2021 | Revisado: 29/11/2021 | Aceito: 30/11/2021 | Publicado: 12/12/2021

Isabel Lima da Silva

ORCID: https://orcid.org/0000-0003-2082-5782

Universidade Ceuma, Brasil

E-mail: bisabellima@gmail.com

Haigle Reckziegel de Sousa

ORCID: https://orcid.org/0000-0002-5803-2289

Universidade Ceuma, Brasil

E-mail: haiglereck3@gmail.com

Felype Hanns Alves de Medeiros

ORCID: https://orcid.org/0000-0001-5026-1995

Universidade Estadual do Pará, Brasil

E-mail: felypehanns@hotmail.com

Keiliane Pinheiro Silva Barros

ORCID: https://orcid.org/0000-0002-0477-9766 Universidade Ceuma, Brasil

E-mail: keilianepinheiromk@gmail.com

Artur de Souza Veras

ORCID: https://orcid.org/0000-0002-6160-8736 Universidade Ceuma, Brasil

E-mail: lasferro1@gmail.com

Patrícia dos Santos Silva Queiroz

ORCID: https://orcid.org/0000-0002-9587-1786

Universidade Ceuma, Brasil

E-mail: patriciasqueiroz@gmail.com

\begin{abstract}
Resumo
Introdução: A experiência do homem durante a trajetória da gestação de sua mulher deve percorrer através da interação estabelecida de forma pessoal, com a gravidez, a companheira e com a família, para essa ligação aconteça é necessário que ele seja incetivando a participar desde o pré-natal. Objetivo: analisar as conduntas de enfermagem frente ao incentivo do homem no pré-natal. Metodologia: A pesquisa em questão trata-se de uma revisão bibliográfica, descritiva e exploratória, sendo fundamentada por artigos publicados em periódicos. A coleta de dados ocorreu no mês de setembro de 2021, os critérios de inclusão foram artigos publicados em língua portuguesa, disponíveis na íntegra de forma gratuita, de janeiro de 2016 a setembro de 2021, no banco de dados da ScieELO e LILACS. Resultados: O presente estudo baseou-se em 10 artigos. Esse estudo trouxe resultados importantes demostrado que para que haja uma boa adesão dos parceiros no acompanhamento do pré-natal é indispensável que seja realizado um planejamento sistematizado das ações, e estes devem incluir o acolhimento, a empatia, comunicação eficaz e ações proativas de educação em saúde fundamentais para que sejam concretizados o vínculo entre profissionais da Atenção Básica e usuários Considerações finais: Conclui-se que o enfermeiro é o principal profissional que tem a responsabilidade de acolher inicialmente o parceiro e conceder a esse indivíduo a oportunidade de se envolver mais diretamente no processo gravídico, tanto nas consultas individuais como nos eventos em grupo, abrindo espaço para que a figura do pai seja protagonista de uma situação nova.
\end{abstract}

Palavras-chave: Pai; Atenção primária a saúde; Enfermagem.

\section{Abstract}

Introduction: The experience of man during the course of the pregnancy of his wife must go through the personal, established interaction with pregnancy, the companion and the family, for this connection to take place it is necessary that he be encouraged to participate since prenatal birth. Objective: to analyze nursing conducts in the face of men's encouragement in prenatal care. Methodology: The survey in question is a bibliographic, descriptive and exploratory review, based on articles published in periodicals. The data collection took place in September 2021, the inclusion criteria were articles published in the Portuguese language, available in full free of charge, from January 2016 to September 2021, in the database of ScieELO and LILACS. Results: This study was based on 10 articles. This study 
has shown important results that in order for the partners to have a good membership in prenatal monitoring, it is essential that systematic planning of actions be carried out, and these should include reception, empathy, Effective communication and proactive actions of education in fundamental health to achieve the link between professionals of Basic Attention and users Final considerations: It is concluded that the nurse is the main professional who has the responsibility of initially welcoming the partner and granting that individual the opportunity to become more directly involved in the gravitational process, both in individual consultations and in group events, making room for the figure of the father to be the protagonist of a new situation.

Keywords: Fathers; Primary health care; Nursing.

\section{Resumen}

Introducción: la experiencia del hombre durante el embarazo de su esposa debe tener una interacción personal y establecida con el embarazo, la pareja y la familia, y para ello es necesario invitarlo a participar desde antes del nacimiento. Objetivo: analizar los canales de enfermería de la estimulación masculina en la atención prenatal. Métodos: la encuesta es una revisión bibliográfica, descriptiva y exploratoria basada en artículos publicados en revistas. La recolección de datos se llevó a cabo en septiembre de 2021 e incluyó artículos publicados en portugués entre enero de 2016 y septiembre de 2021 en las bases de datos scieleo y lillacs, totalmente gratuitos. Resultados: Este estudio se basa en 10 artículos. El estudio demostró que los resultados importantes eran que, para que las parejas tuvieran una buena membresía en la vigilancia prenatal, era necesario un plan de acción sistemático que incluyera la aceptación, la empatía, Comunicación efectiva y acción positiva en la educación básica en salud para lograr la conexión entre el profesional y el usuario de la atención básica. Consideraciones finales: la conclusión es que el enfermero es el profesional principal y tiene la responsabilidad de dar la bienvenida a la pareja y a la persona en primer lugar Tener la oportunidad de participar más directamente en el proceso gravitacional, ya sea en la negociación individual o en las actividades colectivas, para que la imagen del padre se convierta en el protagonista de la nueva situación.

Palabras clave: Padre; Atención primaria de salud; Enfermería.

\section{Introdução}

A atenção materno-infantil é considerada um segmento prioritário na concepção do Ministério da Saúde, sendo determinado como foco principal o acolhimento do pré-natal desde o início da gestação. É sabido que nesse período, a mulher se mostra com maior sensibilidade diante dos acontecimentos intrínsecos e extrínsecos à gravidez, necessitado ter um olhar diferenciado sobre a mulher durante essa trajetória gestacional. As alterações físicas e emocionais inerentes ao seu estado acabam despertando algumas necessidades que, para serem atendidas, necessita de participação e compreensão de pessoas de seu convívio, e nesse caso o companheiro é figura importante nesse processo. (Ferreira et al., 2016) (Oliveira et al., 2009).

Como a gestante, na maioria das vezes, segue as consultas de pré-natal de maneira solitária, consequentemente acaba sendo a única responsável por aderir ao serviço, às orientações e aos exames (Silva et al., 2019). A experiência do homem durante essa trajetória da gestação de sua mulher deve percorrer através da interação estabelecida de forma pessoal, com a gravidez, a companheira e com a família. E, para destacar o papel do homem nesse contexto atualmente é preciso que se leve em consideração alguns elementos que fazem parte da sua concepção e vivência sobre a paternidade, podendo destacar de início a questão do gênero, os modelos transgeracionais e a coexistência de novas demandas sociais (Ferreira et al., 2016).

De forma generalizada, as circunstâncias que estão envolvidas com o universo masculino são uniformalizadas com a figura do provedor, cuidador e chefe da família (Mendes \& Santos, 2019). Dessa forma, a inserção do homem dentro de espaço do pré-natal é necessário de forma que ele possa vivenciar e ter a experiência de acompanhar o crescimento do filho desde a concepção ao seu nascimento (Ferreira et al., 2016)

Diante disso, O Ministério da Saúde discorre sobre diversas etapas para um pré-natal de qualidade, entre eles está justamente o direito do parceiro de também receber uma atenção profissional quanto ao seu cuidado, e isto inclui informações, antes, durante e depois da gestação (Ferreira et al., 2016).

Nesse cenário, é de suma importância uma assistência adequada da enfermagem na questão da prevenção de possíveis morbidades e diminuição da mortalidade desse público. Além disso, essas ações promovem o fortalecimento do vínculo tanto 
com a parceira como com seu filho para que gestante se sinta cada vez mais segura diante do seu momento de fragilidade física e emocional (Lima et al., 2016).

Diante disso, foi aprovada e sancionada a Lei $\mathrm{n}^{\circ} 11.108$, em abril de 2005, que contempla a permissão da presença de um acompanhante escolhido pela parturiente durante todo o período de trabalho de parto, parto e pós-parto imediato. Além disso, a Portaria $\mathrm{n}^{\mathrm{o}} 1.459$, que institui a Rede Cegonha, com a preocupação de assegurar à mulher e à criança uma assistência mais humanizada, concorda com a acompanhante de escolha da parturiente, durante o acolhimento (Holanda et al., 2018).

Nos últimos anos, diversos fatores têm contribuído para que ocorra uma mudança de comportamento entre o companheiro e a companheira, um exemplo disso é a inserção da mulher no mercado de trabalho, que com essa mudança passa a exigir um maior envolvimento seja durante a gestação, ou ainda no cuidado com a criança recém-nascida, sendo que as tarefas são distribúidas por iguais, uma sobrecarga das menor (Lima et al., 2016).

Todavia, por mais que pareça simples de estender a assistência pré-natal ao parceiro da gestante, os serviços de saúde parecem ainda não estar preparados para realizar essa inserção, pois em alguns casos, não ocorre a orientação e nem essa buscativa dos conjugê para que participe de fato desse processo (Ferreira et al., 2018).

Diante do exposto, a motivação para a realização é consequentemente a escolha dessa temática consiste exatamente em procurar o impacto da participação do homem no pré-natal é importante, visando os indicativos de melhorar o desenvolvimento da família envolvida como um todo, da saúde do casal e principalmente do bebê. Assim, a problemática do estudo consiste em quais são as condutas da enfermagem no incentivo a vivencia do homem no pré natal? Sendo o objetivo geral analisar as conduntas de enfermagem frente ao incentivo do homem no pré natal.

\section{Metodologia}

Em relação aos fins, a pesquisa foi exploratória e descritiva. Esse primeiro tipo de pesquisa tem por finalidade proporcionar maior familiaridade com o problema, com vistas de torná-lo mais explícito, buscando maior familiaridade com o problema e buscando visualizar as possíveis soluções para ele:

A grande maioria dessas pesquisas envolve: (a) levantamento bibliográfico; (b) entrevistas com pessoas que tiveram experiências práticas com o problema pesquisado; e (c) análise de exemplos que estimulem a compreensão (GIL, 2007, p. 41).

Além disso, a pesquisa exploratória, para Lakatos e Marconi (2010, p. 171) "os estudos exploratório-descritivo combinados tem por objetivos descrever completamente determinado fenômeno, como, por exemplo, o estudo de um caso para o qual são realizadas análises empíricas e teóricas".

Conforme entendimento de Silva e Menezes (2001, p. 21):

A pesquisa descritiva visa descrever as características de determinada população ou fenômeno ou o estabelecimento de relações entre variáveis. Envolve o uso de técnicas padronizadas de coleta de dados: questionário e observação sistemática. Assume, em geral, a forma de levantamento.

Portanto, a pesquisa busca sempre a melhor aproximação do pesquisador com a situação real dos problemas, além disso, consiste na compreensão de maneira menos totalitária, fragmentando as opiniões apresentadas e objetivando a perspectiva da ótica dos atores que estão envolvidos no processo.

Em relação aos meios, foi aplicado para este estudo os procedimentos de pesquisa bibliográfica. Assim, publicações nacionais, em livros, artigos de autoria de profissionais de enfermagem e revistas fizeram parte da construção desse material: 
A pesquisa bibliográfica é feita a partir do levantamento de referências teóricas já analisadas, e publicadas por meios escritos e eletrônicos, como livros, artigos científicos, páginas de web sites. Qualquer trabalho científico inicia-se com uma pesquisa bibliográfica, que permite ao pesquisador conhecer o que já se estudou sobre o assunto. Existem, porém, pesquisas científicas que se baseiam unicamente na pesquisa bibliográfica, procurando referências teóricas publicadas com o objetivo de recolher informações ou conhecimentos prévios sobre o problema a respeito do qual se procura a resposta (Fonseca, 2002, p. 32)

Assim, é perceptível que, apesar de todo o esforço para levantar os pontos de vista dos atores sociais envolvidos no processo ao qual a pesquisa se propõe a estudar, tem-se que o embasamento teórico através do levantamento bibliográfico, se tornando o alicerce para a compreensão da pesquisa.

Portanto, os critérios de inclusão foram: artigos publicados em língua portuguesa, disponíveis na íntegra de forma gratuita, de janeiro de 2016 a setembro de 2021, com os descritores buscados no Descritores em Saúde (DECs) em portugues e inglês Pai "Father", Enfermagem "Nursing", Cuidado Pré-Natal "Prenatal Care", Os critérios de exclusão foram artigos inferiores ao ano de 2015 e artigos não condizentes com o tema.

A coleta de dados foi realizada de fevererio a março de 2021, sendo utilizados as seguintes bases de dados: LILACS - Literatura Latino-Americana em Ciências de Saúde e SCIELO - Scientific Electronic Library. Para seleção inicial, encontrou-se 95 artigos, através da leitura detalhada e análise dos critérios, obteve-se 10 artigos para compor o artigo final como mostra a Figura 1:

Figura 1 - Fluxograma do processo de seleção dos artigos da presente revisão, 2021

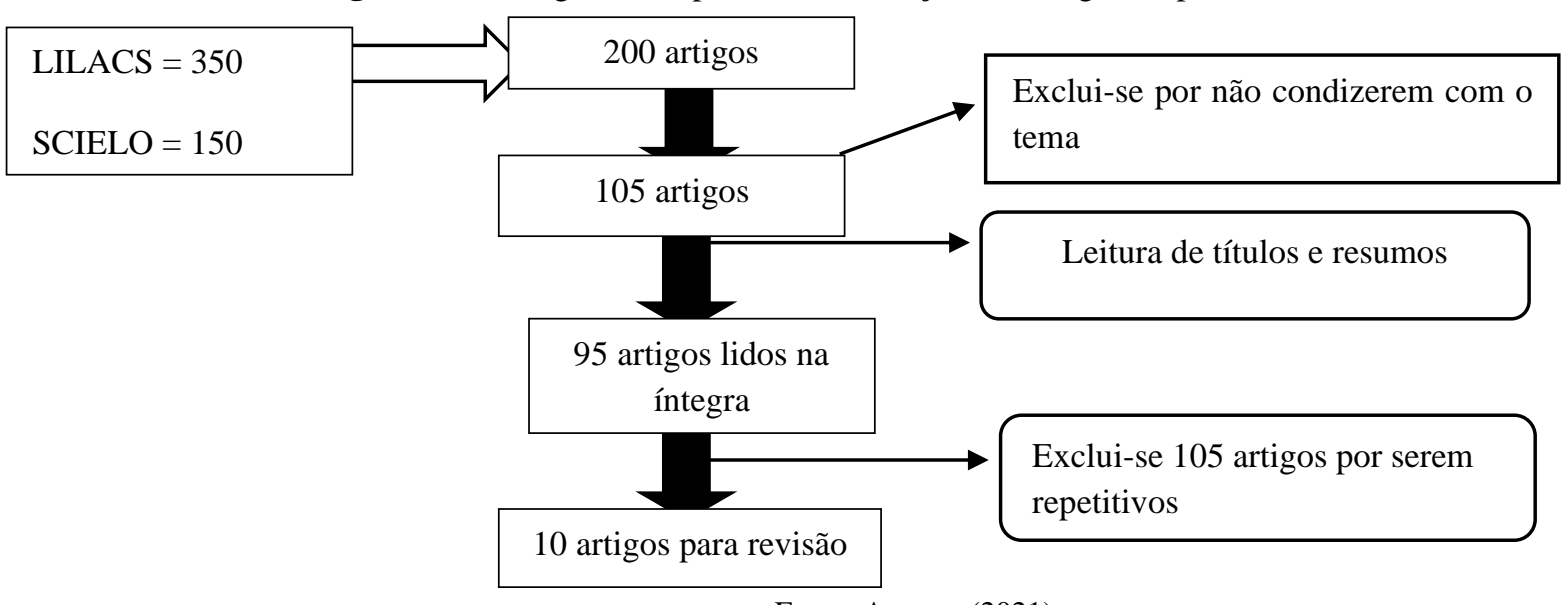

Fonte: Autores (2021).

\section{Resultados e Discussão}

A amostra final desta revisão foi constituída por 10 artigos científicos, selecionados pelos critérios de inclusão previamente estabelecidos. O Quadro 1 representa as especificações de cada um dos artigos, distribuídos por primeiro autor, título, ano e base de dados: 
Quadro 1 - Relação dos estudos selecionados quanto ao ano, periódico, autores e título entre 2015 e 2020.

\begin{tabular}{|c|c|c|c|}
\hline PRIMEIRO AUTOR & TÍTULO & ANO & BASE DE DADOS \\
\hline $\begin{array}{l}\text { Selma Silva de Souza } \\
\text { Bonim }\end{array}$ & $\begin{array}{l}\text { A importância da participação do pai no } \\
\text { acompanhamento do pré-natal }\end{array}$ & 2020 & SCIELO \\
\hline $\begin{array}{l}\text { Layres Canuta Cardoso } \\
\text { Climaco }\end{array}$ & $\begin{array}{l}\text { Pré-natal masculino: um relato de experiência } \\
\text { no contexto da educação em saúde }\end{array}$ & 2016 & LILACS \\
\hline Gabriela Sofia Henz & A inclusão paterna durante o pré-natal & 2017 & LILACS \\
\hline Julien Caju Marques & $\begin{array}{l}\text { O olhar da enfermagem sobre o pré-natal } \\
\text { masculino: possibilidades e desafios }\end{array}$ & 2020 & SCIELO \\
\hline $\begin{array}{l}\text { Rosa Maria Santos } \\
\text { Medeiros }\end{array}$ & $\begin{array}{l}\text { Pré natal masculino: desafios na prática de } \\
\text { enfermagem na atenção básica à saúde }\end{array}$ & 2019 & SCIELO \\
\hline Silma Costa Mendes & $\begin{array}{l}\text { Pré-natal masculino: a importância da } \\
\text { participação do pai nas consultas de pré-natal }\end{array}$ & 2019 & SCIELO \\
\hline Ana Emília Oliveira & $\begin{array}{l}\text { Emergências obstétricas: características de } \\
\text { casos atendidos por serviço móvel de urgência }\end{array}$ & 2020 & SCIELO \\
\hline Wilmara Cardoso Silva & $\begin{array}{l}\text { Pré-natal do parceiro: desafios para o } \\
\text { Enfermeiro. }\end{array}$ & 2020 & SCIELO \\
\hline Rosyanne da Silva & $\begin{array}{l}\text { Pré-natal do parceiro: uma análise a partir da } \\
\text { perspectiva da gestante }\end{array}$ & 2020 & SCIELO \\
\hline Lucas Augusto Santana & $\begin{array}{c}\text { A participação do parceiro na rotina pré-natal } \\
\text { da mulher gestante: estudo em uma unidade } \\
\text { básica de saúde }\end{array}$ & 2020 & LILACS \\
\hline
\end{tabular}

Fonte: Autores (2021).

A visão que a grande maioria atribui ao pai é a função daquele como provedor financeiro, mostrando o machismo estrutural presente na sociedade, diante desse contexto, o Brasil vem procurando mudar essa ideia, ou seja, procura de forma eficiente incluir o homem no processo família (Henz, Medeiros \& Salvadori ,2017) (Silva et al, 2020).

Segundo Medeiros (2017) a figura paterna vem sendo ainda excluída pelos profissionais de saúde, mas isso não esta relacionado somente a essa questão, ao contrário, pode ocorrer pela falta de estrutura física e capacitação das equipes no momento do atendimento a esse público. Com o passar dos anos, os homens têm sempre se destacado na agenda de saúde, ou seja, sendo passíveis de estudos nas áreas de sistemas de informação epidemiológica e produção científica e políticas públicas, despertando uma atenção especial para proporcionar o apoio necessário para esse gênero (Climaco et al., 2020).

Sendo um desses núcleos, as ações que possam captar o homem no pré-natal, podendo ser elas a identificação dos fatores que influencie no ciclo gravídico, sendo eles ambientais, sociais ou econômicos, assim, a consulta pré-natal deve ser realizada durante os três trimestres, com intuito de uma avaliação das condições materna e fetal. A prevenção ou pelo menos diagnóstico precoce dos sinais anormais, seguida por tratamento imediato e efetivo, evitará muitas complicações associadas a parturição, não somente durante o período anteparto, como também durante o trabalho de parto, o nascimento e o puerpério. Portanto, a assistência anteparto é essencialmente uma assistência preventiva para a mãe e o feto (Silva et al., 2020) (Mendes

Dessa forma, a participação do pai precisa fazer parte de todos os momentos que envolvem o nascimento da criança, principalmente na saúde, onde também é necessário que o profissional consiga estimular esse processo que ainda encontra alguns entraves.

No Brasil a lei $\mathrm{n}^{\circ} 11.108 / 2005$ concede as parturientes o direito da presença do acompanhante durante o trabalho de parto, pré-parto e pós-parto, mas essa informação precisa ser lembrada e informada sempre aos pais desde o primeiro dia de acompanhamento pré-natal (Silva, Oliveira \& Saraiva, 2020). 
Ao acompanhar sua parceira nas consultas de pré-natal, o pai acaba experimentando a paternidade por um outro ângulo e assim exercendo sua função, além de tornar o processo gestacional para a mãe bem mais saudável e prazeroso (Henz, Medeiros \& Salvadori, 2017) (Marques et al, 2020).

Nesse contexto, os autores apenas confirmam e reforçam que a realização do pré-natal não é responsabilidade única da mulher, a participação do parceiro precisa ser mais ativa, entretanto para conter a desigualdade que predomina no gênero masculino, constitui-se para prática de uma paternidade ativa e cuidadosa antes, durante e depois do nascimento, ficou normatizado e preconizado pelo Ministério da Saúde o pré-natal do homem. Um desses acompanhamentos são a realização de exames laboratorias da parte paterna, é visto que as mães tem esse acompanhamento de saúde, entretanto, pesquisas recentes demostram que fatores biológicos paternos são de fundamental importância para monitoramente das condições de saúde como rastreamento de hemoglopatias, infecções sexualmente transmissiveis (ISTs) não tratada, toxoplasmose, dislipedemia, hepatite B e diabetes melitus (Henz, Medeiros \& Salvadori, 2017) (Silva et al., 2020).

É de suma necessidade que após a confirmação da gestação, o enfermeiro, já comece na primeira consulta a influenciar que o pai seja presente no acompanhamento, mostrando como será benéfico para a mulher e a para o bebe a presença do pai. O profissional deve tomar uma postura que transpareça o acolhimento, incentivando a presença nas próximas consultas, apontando que será um momento em que o pai pode conversar melhor e tirar todas as suas dúvidas, além de compreender para que serve e qual a importância de cada exame solicitado (Silva, Oliveira \& Saraiva, 2020).

A enfermagem deve prestar bastante atenção em relação a situação vacinal do pai/parceiro, e é exatamente no momento da consulta que ele tem essa oportunidade de atualizar o cartão dessa figura paterna, sempre procurando explicar a relevância desses cuidados. É importante que seja criado e estabelecido esse vínculo entre enfermeiro e o pai, deixando ambos dos parceiros mais à vontade (Holanda, 2018).

Diante disso, quando se faz essa inclusão da participação da paternidade durante o pré-natal o enfermeiro percebe que ela influência bastante e positivamente em relação à convivência familiar, agregando um ambiente mais harmoniosos e consequentemente quando a criança nascer terá mais amor e tranquilidade para se desenvolver, pois quando a mulher sente mais segura (Cardoso et al., 2018).

Portanto, a atuação do enfermeiro atuando como membro importante da equipe de saúde, além de ser responsável pelo atendimento das consultas pré-natais na atenção básica consegue proporcionar situações para melhorar o acolhimento desse homem/pai na unidade de saúde, proporcionando-lhes condições para interagir juntamente com a gestante/companheira no processo gravídico (Medeiros, 2019).

Ressalta-se, no entanto, que os pais, mesmo que jovens e adolescentes, ainda necessitam de uma atenção mais estratégica e direcionada, levando em consideração os aspectos como a baixa idade que acabam aumentando as dificuldades em relação ao entendimento quanto ao planejamento familiar e a importância de um acompanhamento sistematizado no que se refere ao cuidado da mãe e do RN, e principalmente como da sua saúde enquanto figura paterna (Oliveira \& Farias, 2020) (Santana, 2020). (Mendes et al., 2019).

Nesse momento é a oportunidade do enfermeiro atuar na educação do escolar com o objetivo de prevenir uma gestação indesejada e que ocasione situações desconfortáveis, orientações quanto a sexualidade e demais assuntos relacionados à saúde são essenciais nesse processo, somente assim pode-se prestar uma assistência de qualidade (Henz, Medeiros \& Salvadori, 2017) (Bonim et al., 2020).

A atuação do parceiro no pré-natal ganha maior visibilidade depois que foram criadas as ações do Ministério da Saúde voltada unicamente para atender a esse objetivo de público: 
Diante disso, a atuação da enfermagem para que realize uma assistência de qualidade se faz presente no Guia do PréNatal do Parceiro, documento estruturado para orientar os Profissionais de Saúde quanto as estratégias de ação para o envolvimento consciente e ativo de homens, adolescentes, jovens adultos e idosos em todas as ações voltadas ao cuidado com a saúde e, ao mesmo tempo, contribuir para a ampliação e a melhoria do acesso e acolhimento desta população aos serviços de saúde, com enfoque na Atenção Básica (Silva, 2020, p. 134).

Nesse contexto, para que haja uma boa adesão dos parceiros no acompanhamento do pré-natal é indispensável que seja realizado um planejamento sistematizado das ações, e estes devem incluir o acolhimento, a empatia, comunicação eficaz e ações proativas de educação em saúde fundamentais para que sejam concretizados o vínculo entre profissionais da Atenção Básica e usuários (Cardoso, et al, 2018).

\section{Considerações Finais}

O que se torna cada vez mais evidente é a necessidade da integração da política do homem a outras diretrizes, pois, a anuência do homem ao Pré-natal acaba proporcionando o combate aos agravos evitáveis e ao alto índice de morbimortalidade, além da capacidade de detectar mais precocemente tratamento de doenças crônico degenerativas e em especial infecções sexualmente transmissíveis.

As condutas da enfermagem ao incentivo do homem no pré natal devem fazer parte da sensibilização da equipe de saúde, mais precisamente por meio dos Agentes Comunitários de Saúde, Enfermeiros e Médicos, considerados ponta do processo também é vital para a conscientização das gestantes e do parceiro durante essas consultas, garantindo assim, a integralidade da assistência ao trinômio mãe/pai/feto, destacando o papel de cada um durante esse processo que exige união e participação dos envolvidos.

$\mathrm{O}$ resultado apresentado nesse estudo destaca a necessidade de implementação de estratégias que possibilitem o entendimento quanto à importância da participação do pai nas orientações e acompanhamento durante todo o processo gestacional, mais precisamente nas consultas de pré-natal, reforçando que a Unidade Básica de Saúde é a principal porta de entrada para um atendimento e orientação necessária para os cuidados na gestação.

Conclui-se que o enfermeiro é o principal profissional que tem a responsabilidade de acolher inicialmente o parceiro e conceder a esse indivíduo a oportunidade de se envolver mais diretamente no processo gravídico, tanto nas consultas individuais como nos eventos em grupo, abrindo espaço para que a figura do pai seja protagonista de uma situação nova.

Consoante a este tema, faz-se necessário também elencar futuros trabalhos acerca do parceiro enquanto acompanhante durante o trabalho de parto, visto que ambos os temas são de extrema importância para as pacientes e são comumente deixados de lado pela comunidade, entretanto, é indispensável pensar na presença do pai não somente durante o pré-natal, mas também ao longo do trabalho de parto.

\section{Referências}

Cardoso, V. E. P. S., Silva Junior, A. J. D., Bonatti, A. F., Santos, G. W. S. D., \& Ribeiro, T. A. N. (2018). A participação do parceiro na rotina pré-natal sob a perspectiva da mulher gestante. Rev. pesqui. cuid. fundam. (Online), 856-862.

Climaco, L. C. C., Vilela, A. B. A., Yarid, S. D., \& Boery, E. N. (2020). Pré-natal masculino: um relato de experiência no contexto da educação em saúde. Enfermagem em Foco, 11(2).

Ferreira, I. S., Fernandes, A. F. C., Lô, K. K. R., Melo, T. P. D., Gomes, A. M. F., \& Andrade, I. S. (2016). Perceptions of pregnant women about the role of partners in prenatal consultations.

Fonseca, J. J. S. (2002). Apostila de metodologia da pesquisa científica. João José Saraiva da Fonseca.

Oliveira, S. C., Ferreira, J. G., da Silva, P. M. P., Ferreira, J. M., de Almeida Seabra, R., \& Fernando, V. C. N. (2009). A participação do homem/pai no acompanhamento da assistência pré-natal. Cogitare Enfermagem, 14(1), 73-78. 
Research, Society and Development, v. 10, n. 16, e243101623666, 2021

(CC BY 4.0) | ISSN 2525-3409 | DOI: http://dx.doi.org/10.33448/rsd-v10i16.23666

Oliveira, A. E. A., \& de Farias, G. M. incentivo ao pré-natal do parceiro na atenção primária em saúde: um relato de experiência.

Souza Bonim, S. S., Andrade, E. X., Nunes, V., \& Looze, J. T. T. A importância da participação do pai no acompanhamento do pré-natal.

Gil, A. C. (2002). Como elaborar projetos de pesquisa (Vol. 4, p. 175). São Paulo: Atlas.

Henz GS, Medeiros CRG \& Salvador M (2017). A inclusão paterna durante o pré-natal. Revista de Enfermagem e Atenção à Saúde [Online]. jan/jun; 6(1): 5266. DOI: DOI: $10.18554 /$ reas.v6i1.2053

Holanda, S. M., Castro, R. C. M. B., Aquin, P. D. S., Pinheiro, A. K. B., Lopes, L. G., \& Martins, E. S. (2018). influência da participação do companheiro no pré-natal: satisfação de primíparas quanto ao apoio no parto1. texto \& Contexto-Enfermagem, 27.

Marconi, M. D. A., \& Lakatos, E. M. (2010). Fundamentos de metodologia científica. 5. ed.-São Paulo: Atlas.

Lopes, G. D. S., Sousa, T. V. D., Freitas, D. D. A., Carvalho Filha, F. S. S., Sá, E. S. D., Vasconcelos, A. C. D., ... \& Moraes Filho, I. M. D. (2021). Os benefícios do pré-natal masculino para a consolidação do trinômio mãe-pai-filho: uma revisão integrativa. Revista de Divulgação Científica Sena Aires, 10(1), 22-38.

Lima, S. R., Wanderley, T. C., \& de Albuquerque Araújo, L. D. C. (2016). O papel da enfermagem na assistência ao pré-natal do parceiro. Revista SaúdeUNG-Ser, 10 (1 ESP), 52 .

Marques, J.C et al. (2020) O olhar da enfermagem sobre o pré-natal masculino: possibilidades e desafios. Temas em Saúde. 1 (1), $324-329$.

Medeiros, R. M. S. D., Coutinho, S. P. M., Maia, A. M. C. D. S., Sousa, A. R. D., Oliveira, M. T., Rosário, C. R. D., \& Passos, N. C. R. (2019). Pré-natal masculino: desafios na prática de enfermagem na atenção básica à saúde. Revista de Divulgação Científica Sena Aires, 8(4), 394-405.

Mendes, S., \& Santos, K. C. (2019). Pré-natal masculino: a importância da participação do pai nas consultas de pré-natal. Enciclopédia biosfera, 16(29).

Santana, L. A., \& da Silva Gonçalves, B. D. (2020). A participação do parceiro na rotina pré-natal da mulher gestante: estudo em uma unidade básica de saúde. Humanidades e tecnologia (FINOM), 20(1), 312-327

Silva, E. L. D. \& Menezes, E. M., \& (2001). Metodologia da pesquisa e elaboração de dissertação. Florianópolis: LED/UFSC.

Silva, W. C., Wanderley, R. R., Markus, G. W. S., Pereira, R. A., do Couto, G. B. F., \& Dias, A. K. (2020). pré-natal do parceiro: desafios para o enfermeiro. Revista Extensão, 4(2), 127-137.

Silva, R., de Oliveira, S. C., \& Saraiva, A. P. C. (2020). Pré-natal do parceiro: uma análise a partir da perspectiva da gestante. Revista Eletrônica Acervo Saúde, 12(12), e4361-e4361. 